psychiatrists and service managers in old age psychiatry who wish to make a robust case for increasing the availability of psychological services to older people.

Elizabeth Sampson MRC Research Fellow and Old Age Psychiatrist, Department of Mental Health Sciences, Royal Free and University College Medical School, Rowland Hill Street, London NW3 2PF, UK. E-mail: e.sampson@medsch.ucl.ac.uk

\section{Psychotherapists as Expert Witnesses: Families at Breaking Point}

By Roger Kennedy. London: Karnac Books. 2005. I80pp. $€ 19.99$ (pb). ISBN 1855753723

Child and adolescent psychiatrists - don't be put off by the title. This is a useful book if you want to improve your skills as an expert witness in family cases, whether or not you present yourself as a psychotherapist. And these days, post-Meadow, all the support possible is necessary. It is not surprising that, on the one hand, courses are oversubscribed and that, on the other, general clinicians are reluctant to get involved in court situations. Within the legal system the stakes for the participants (especially the children and parents) are high, the system is adversarial, outcomes uncertain and feedback from 'results' hard to come by. In addition, disgruntled parents, often supported by organised pressure groups, may push reporting to the General

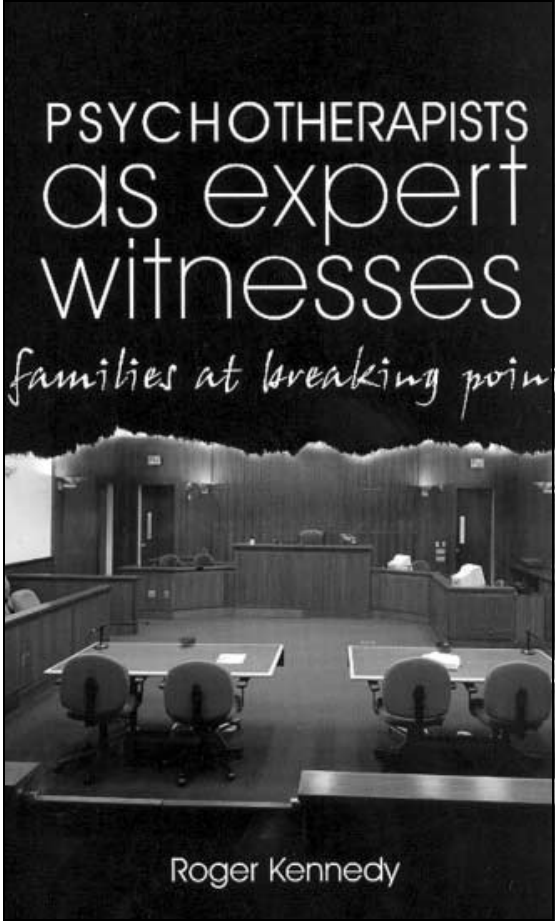

Medical Council of doctors whose actions or recommendations they dislike.

The interest of the child is, of course, primary. Kennedy uses amended real-life vignettes to illustrate how he assesses this, which is helpful. He often judges parental capacity for change by willingness to admit some responsibility for what has gone before. Although we might prefer, at a time of evidence-based medicine, general results from research or even case audit, experts have to depend for the quality of their judgements quite considerably on their own service and therapeutic experiences. Here Roger Kennedy's connection with the Cassel Hospital Family Service is clearly of great value, but is available to few professionals. Kennedy makes the point that recommendations need to be formed in the context of access to therapeutic resources and the willingness of the relevant authorities to fund them. The role as negotiator and mediator outside of the courtroom is also pivotal.

The report of the Royal College of Pathologists \& Royal College of Paediatrics and Child Health (2004), although on a somewhat different topic, recommends that doctors should not confuse or combine the role of professional witness - testifying as observer of a clinical/treatment process with that of the expert witness, who should be independent. A psychiatrist hopes to be 'therapeutic' even in assessment but in court will need at the very least to clarify in which role he or she is acting.

Royal College of Pathologists \& Royal College of Paediatrics and Child Health (2004). Sudden Unexpected Death in Infancy: A Multi-agency Protocol for Care and Investigation. London: Royal College of Pathologists \& Royal College of Paediatrics and Child Health.

Fiona Subotsky Emeritus Consultant Child and Adolescent Psychiatrist, South London and Maudsley NHS Trust, The Belgrave Department, King's College Hospital, Denmark Hill, London SE5 9RS, UK. E-mail: subotsky@clara.co.uk 